\title{
Multi-pronged approach uncovers sJIA mechanisms
}

New research demonstrates how an integrative analysis of multidimensional data can provide insights into the immune alterations that underlie complex diseases such as systemic juvenile idiopathic arthritis (sJIA). By using a blood stimulation assay to capture gene expression profiles, cell subset-specific activation status and secreted protein responses, Cepika et al. found alterations in cytokine production and activation of leukocyte subsets in blood from patients with sJIA.

Although sJIA is known to be driven by IL-1 and can be treated with therapies that block IL-1 or IL-6, the aetiology of this autoinflamma-

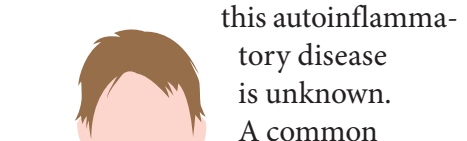

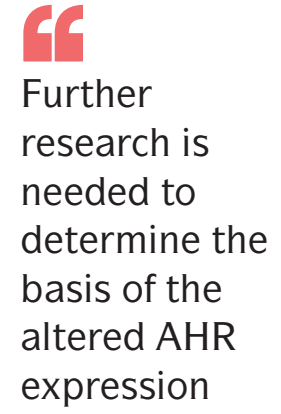

5 approach for examining the state of the immune system in systemic autoimmune and inflammatory diseases is to analyse the ex vivo transcriptional profile of circulating leukocytes; however, as the study's corresponding author Virginia Pascual explains, the transcriptional profile of patients with sJIA largely overlaps with that of several other infectious and inflammatory diseases. "To reveal potential immune alterations more specific to sJIA, we decided to 'exercise' the immune system by stimulating leukocytes ... with multiple pro-inflammatory ligands in vitro, assuming that differences between patients and healthy controls might be found in inducible pathways," says Pascual.

The researchers then characterized the responses using cDNA microarrays, multicolour flow cytometry and multiplex cytokine profiling. "Analytical integration of the results from these three platforms enabled us to link the activation of specific cell subsets and skewing of cytokine production to groups of functionally related genes," Pascual reports.

The researchers focused their studies on cells obtained from patients with sJIA in remission, thereby avoiding potential confounding of the experimental results by disease activity level or medication. In leukocytes from these patients, the assay revealed dysregulated transcriptional and cellular responses to stimulation with Toll-like receptor
4 (TLR4), TLR7 and TLR8 ligands, compared with healthy controls.

Further examination of sJIA monocytes isolated from the patients in remission showed that these cells had increased production (though not secretion) of IL- $1 \beta$ and decreased expression of the gene encoding aryl hydrocarbon receptor (AHR), a regulator of IL- $1 \beta$-mediated inflammation and macrophage polarization. After confirming in an independent cohort that baseline expression of AHR is lower in sJIA monocytes than in those from healthy controls, Cepika et al. showed that sJIA monocytes predominantly differentiate into macrophages, rather than dendritic cells, in vitro. "This bias toward macrophage differentiation might represent a link between sJIA and macrophage activation syndrome (MAS), a very serious and potentially lethal complication of the disease," Pascual explains.

Further research is needed to determine the basis of the altered AHR expression in the cells of patients with sJIA. "We also need to understand how alterations in the AHR pathway might lead to IL-1 dysregulation and, eventually, MAS," says Pascual. "Ideally, understanding the exact mechanism of IL-1 dysregulation in sJIA could lead to the identification of at-risk children before they develop the disease, and provide the tools to prevent its onset and flares," she concludes.

Sarah Onuora

ORIGINAL ARTICLE Cepika, A. M. et al. A multidimensional blood stimulation assay reveals immune alterations underlying systemic juvenile idiopathic arthritis.J. Exp. Med. http://dx.doi. org/10.1084/jem.20170412 (2017) 